\title{
Editorial
}

\section{Nano- and Biomaterials for Sustainable Development}

\author{
An-Ya Lo, ${ }^{1}$ Chuan Wang, ${ }^{2}$ Wei Hsuan Hung, ${ }^{3}$ Anmin Zheng, ${ }^{4}$ and Biswarup Sen ${ }^{5}$ \\ ${ }^{1}$ Department of Chemical and Materials Engineering, National Chin-Yi University of Technology, Taichung 41170, Taiwan \\ ${ }^{2}$ Electrical and Computer Engineering, Michigan State University, East Lansing, MI 48824-1226, USA \\ ${ }^{3}$ Department of Materials Science and Engineering, Feng Chia University, Taichung 40724, Taiwan \\ ${ }^{4}$ Wuhan Institute of Physics and Mathematics, Chinese Academy of Sciences, Wuhan 430071, China \\ ${ }^{5}$ Amity Institute of Biotechnology, Amity University Haryana, Gurgaon 122413, India \\ Correspondence should be addressed to An-Ya Lo; a.y.lo1125@gmail.com and Biswarup Sen; bisen0102@gmail.com
}

Received 18 April 2015; Accepted 18 April 2015

Copyright (C) 2015 An-Ya Lo et al. This is an open access article distributed under the Creative Commons Attribution License, which permits unrestricted use, distribution, and reproduction in any medium, provided the original work is properly cited.

With climate change and energy scarcity, the term "sustainability" has become the major challenge of the 21st century. Researches on clean and renewable energy are vital to break through this problem. Besides, any manners that help to reduce energy and resource consumption, enhance energy efficiency, and minimize the use and generation of hazardous substances are considered "sustainable" as well. Nano- and biorelated technologies have recently received immense attention for their applications on sustainable development. Therefore, there are many research efforts required to be addressed on the potential improvement of materials optical properties, noble metal nanoparticle preparation and properties, and biosynthesis, especially for organic and biocompatible ones, to achieve the upcoming challenges in the advanced applications. This special issue contains six papers; most of them involve both bio- and nanoengineering and technologies.

The paper "Biocomposite of Cassava Starch Reinforced with Cellulose Pulp Fibers Modified with Deposition of Silica $\left(\mathrm{SiO}_{2}\right)$ Nanoparticles" authored by J. Raabe et al. studies the effect of $\mathrm{SiO}_{2}$ nanoparticle modification on the thermal stability, mechanical property, and hygroscopicity of cellulose fibers and their composites with starch. Results show improved tensile strength and reduced moisture absorption in the aforementioned reinforced starch, demonstrating the potential of such biodegradable material as replacement for conventionally used synthetic polymers.

The study titled "Organic Systems and Their Photorefractive Properties under the Nano- and Biostructuration:
Scientific View and Sustainable Development" demonstrates a potential impact of using organic systems such as DNA for modifying photorefractive properties of optoelectronic and biomedical application. The improvement of the photorefractiveness of the organic structures has been achieved by using nano- and biostructuring process, resulting in the changes of laser-induced refractive index and the rotation of the plane of polarization of light based on the model polyimide (PI) photosensitive donor-acceptor matrix and the nematic liquid crystal (NLC) compounds.

In the paper "Sustainable Nanopozzolan Modified Cement (NPMC): Characterizations and Morphology of Calcium Silicate Hydrate (C-S-H) during Hydration," N. M. Sutan et al. have investigated the microstructural differences between two types of cement replacements as nanopozzolan modified cement (NPMC) and estimated the relationship between the pozzolanic reactivity and their physical/ mechanical properties. It is found that the optimum dosage for MS and NS as the replacement in cement paste is $10 \%$ and $5 \%$, respectively, which is due to the presence of the highest intensity in C-S-H. At this condition, the mechanical properties of NPMC in terms of compressive strength were improved by $67.8 \%$. The synergies between MS and NS were well characterized by the XRD, FTIR, EDS spectroscopy analysis, and SEM morphology results that showed evidence of MS-NS interaction with the evidence of decreasing $\mathrm{CH}$ and increasing C-S-H.

The paper, titled "Biosynthesis of Anisotropic Silver Nanoparticles by Bhargavaea indica and Their Synergistic 
Effect with Antibiotics against Pathogenic Microorganisms" authored by P. Singh et al., investigated the possibility to synthesize silver nanoparticles with various shapes by bacterial strain DC1. Without any extra reducing and capping agent, the strain Bhargavaea indica DC1 provides a rapid, ecofriendly, and economical route for the mass production of Ag-NPs. They indicate that the proposed method has also the potential to endow Ag-NPs with antimicrobial activity and also enhance the antimicrobial activity of antibiotics against pathogenic microorganisms, such as Vibrio parahaemolyticus, Salmonella enterica, Staphylococcus aureus, Bacillus anthracis, Bacillus cereus, Escherichia coli, and Candida albicans.

In the article entitled "Study of the Plasmon Energy Transfer Processes in Dye Sensitized Solar Cells" authors successfully demonstrated plasmonic enhancement of dye sensitized solar cells with up to $45 \%$ improvement in the cell power conversion efficiency by simply integrating a layer of island-like plasmonic gold nanoparticles with an $\mathrm{N}_{119} / \mathrm{TiO}_{2}$ DSSC. Moreover, the further plasmonic energy transfer mechanism is also investigated and discussed in plasmonic DSSC system; this significant outcome would potentially benefit on achieving high solar energy conversion efficiency in DSSC system.

L. P. Lukhele et al. report the acute toxicity of doublewalled carbon nanotubes (DWCNTs) to three aquatic organisms (algae, macroinvertebrate, and fish) of different trophic levels. The study of the effect of carbon nanotubes (CNTs) on the water bodies after consumption could indicate the longterm influence of the CNTs and their fate in the environment. The authors show different $\mathrm{LC}_{50} / \mathrm{EC}_{50}$ of DWCNTs for the three organisms, and humic acid and ionic strength of water were found to affect the toxicity levels. The acute toxicity of DWCNTs was dependent on their aggregation state in the aquatic system. Humic acid reduced the hydrodynamic size of the DWCNTs, whereas cations increased the size, thereby lowering the probability of interaction of DWCNTs with organisms.

\section{Acknowledgments}

The guest editors wish to thank all the authors for their valuable contribution to the special issue. We also express our sincere gratitude to all expert reviewers for their valuable time and effort to bring out and maintain the quality of the special issue.

An-Ya Lo Chuan Wang Wei Hsuan Hung Anmin Zheng Biswarup Sen 

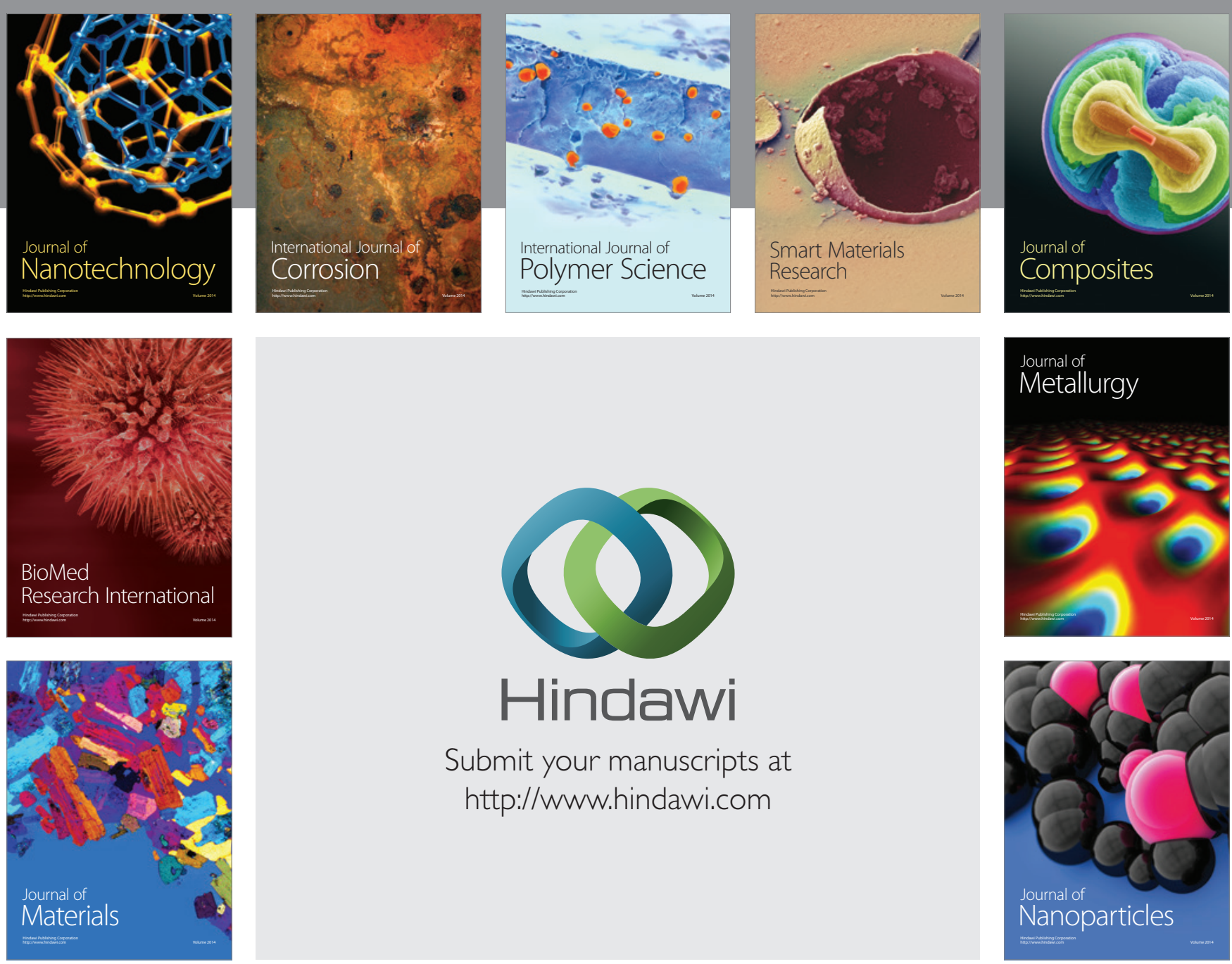

Submit your manuscripts at http://www.hindawi.com
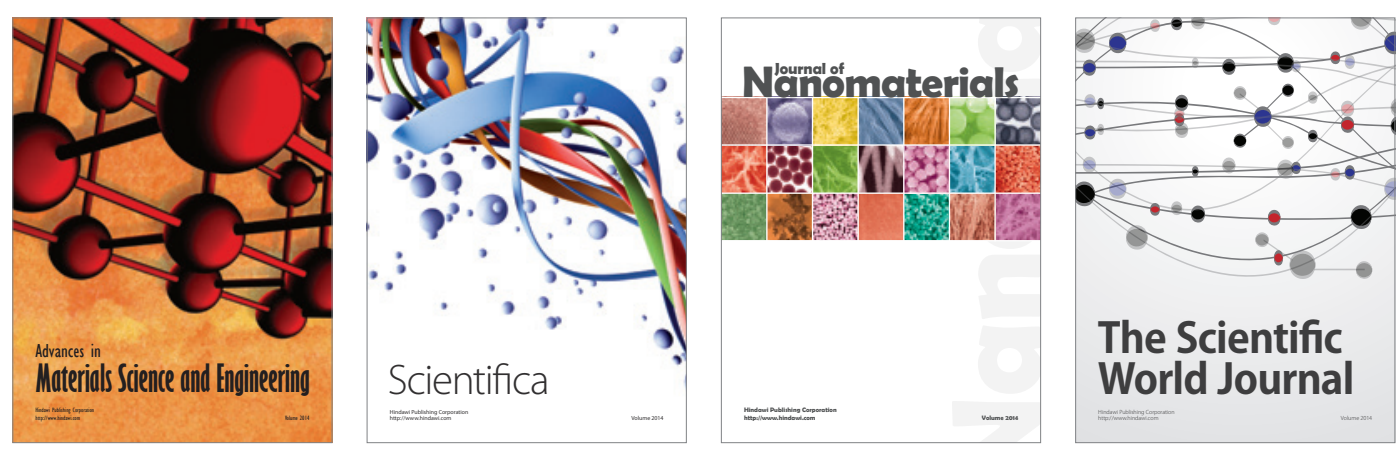

\section{The Scientific World Journal}
